\title{
Preoperative assessment of endometrial cancer
}

\author{
Péter Török, Zoárd Krasznai, Szabolcs Molnár, Rudolf Lampé, Attila Jakab \\ Department of Obstetrics and Gynecology, Faculty of Medicine, University of Debrecen, Debrecen, Hungary \\ Contributions: (I) Conception and design: P Török; (II) Administrative support: P Török, Z Krasznai, S Molnár, A Jakab; (III) Provision of study \\ materials or patients: P Török, Z Krasznai, S Molnár, A Jakab; (IV) Collection and assembly of data: P Török, Z Krasznai, S Molnár, A Jakab; (V) \\ Data analysis and interpretation: P Török, Z Krasznai, S Molnár, A Jakab; (VI) Manuscript writing: All authors; (VII) Final approval of manuscript: \\ All authors. \\ Correspondence to: Dr. Péter Török. Department of Obstetrics and Gynecology, Faculty of Medicine, University of Debrecen, 4032 Debrecen, \\ Nagyerdei krt. 98, Hungary. Email: petertorokdr@gmail.com.
}

\begin{abstract}
Endometrial cancer (EC), the most common among gynaecological malignancies occurs predominantly after the menopause. The diagnosis is most commonly (in about 75-80\%) set up at early stage when surgical therapy and if necessary postoperative radiotherapy results in an excellent prognosis, with a 90-95\% 5-year overall survival (OS) and a locoregional recurrence rates of 4-8\%. Accurate preoperative assessment of the lymph nodes would ideally identify those patients with advanced stage disease, who might benefit from more extensive surgical procedures and adjuvant therapies. magnetic resonance imaging (MRI), hysteroscopic excisional biopsy (HEB) and high resolution 2D or 3D ultrasound performed by expert operator are considered to add valuable information for preoperative staging of EC. The use of biomarkers could be beneficial in decreasing inter-observer variability between the histology of the diagnostic specimen and the final operative sample, as well as to avoid overtreatment in a part of the high-grade tumors with excellent prognosis. The goal of surgical management of EC is to remove the primary tumor and to identify definite prognostic factors to determine whether adjuvant therapy is required. Extended surgery, including para-aortic lymphadenectomy has a significant morbidity, and with the future selection of cases it can be safely avoided, we can decrease complications without compromising oncological safety. In the future, the importance of hysteroscopy guided sampling may increase to gain a representative sample for biomarker detection.
\end{abstract}

Keywords: Endometrial cancer (EC); hysteroscopy; magnetic resonance imaging (MRI); staging; ultrasound

Submitted May 12, 2020. Accepted for publication Jul 06, 2020.

doi: $10.21037 /$ tcr-20-2068

View this article at: http://dx.doi.org/10.21037/tcr-20-2068

\section{Introduction}

Endometrial cancer (EC), as the most common gynaecological malignancies comprises more than one in 20 female cancers globally and occurs predominantly after the menopause (1). The diagnosis is most commonly set up at early stage, $75-80 \%$ of the disease is detected at International Federation of Gynaecology and Obstetrics (FIGO) stage I. The results of postmenopausal patients are even better, whose cancers are strongly associated with abnormal uterine bleeding (2). Surgical therapy and if necessary postoperative radiotherapy results in excellent prognosis, with 90-95\% 5-year overall survival (OS) and locoregional recurrence rates of 4-8\% (3). In advanced cases OS falling continuously till $20 \%$ at EC stage 4 . Prognostic factors are presence of lymph node involvement, histological type of the tumor (type I and type II cancers), cervical stroma invasion, the depth of myometrial invasion (MI) and the grade of the tumor (4). Besides the invasion of the cervical stroma, according to a recent study, the presence of (atypical) glandular cells (GC) in the preoperative cervical smear can be a predictor of local recurrence. The GC-s are more frequently detected in patients with grade 3 and non-endometrioid tumors (5). Incidence of pelvic 
node involvement differs from 0 up to $20-60 \%$, paraaortic 4 to $10-30 \%$ depending on the histological type and grade of the tumor, as well as the depth of MI (6). According to ESGO guidelines, and ESMO-ESGOESTRO consensus conference, preoperative assessment of the tumor is essential: this include imaging [pelvic magnetic resonance imaging (MRI), abdominal and chest CT], and preoperative histology. The surgical management should be based on these findings. PET-CT can also be a useful tool in detecting retroperitoneal metastases, although it has a limited sensitivity in intermediate and high-risk early EC, so it cannot be used as an alternative tool for surgical staging (7). The standard operation is total hysterectomy with bilateral salpingo-oophorectomy (BSO), preferably via endoscopic procedures in low risk cases. Systemic pelvic and paraaortic lymphadenectomy is recommended in high risk and can be considered in intermediate risk cases as part of surgical staging $(8,9)$. Sentinel lymph node biopsy can be performed as well and-as based on the results of the FIRES multicentre prospective study-is equally effective in the detection of metastatic nodes with a sensitivity of $97.2 \%$, and a negative predictive value (NPV) of $99.6 \%$ (10). The SHREC trial (Sentinel lymph node detection in High Risk Endometrial Cancer) had similar results: the sentinel lymph node-indocyanine green (ICG) algorithm had a sensitivity of $98 \%$ and a NPV of $99.5 \%$ (11). It is important to note that the sensitivity of sentinel lymph node detection largely relies on the proper method (preferably ICG as in the upper cases, or radiocolloid/Tc $99 \mathrm{~m} /$ and color dye together), and surgical experience to be able to track these nodes, but as a result it provides the same oncological safety with much less morbidity compared to systemic lymph node staging. It is very important to stick to the SLN algorithm and perform a side specific complete lymphadenectomy in case of unsuccessful SLN detection or any suspicious enlarged nodes. It is no longer considered an experimental technique, and is part of the international guidelines (12). Lymphadenectomy plays role in identifying patients who require adjuvant chemotherapy and radiotherapy (13). Pelvic washings are no longer required for surgical staging, since the presence of cancer cells in the peritoneal cavity is a poor prognostic factor (14).

The goal of surgical management of EC is to remove the primary tumor and to identify definite prognostic factors to determine whether adjuvant therapy is required. The type of surgical approach is planned based on the preoperative impression of stage, which is based on the pretreatment evaluation (15). Randomized trials have demonstrated that minimally invasive approaches to EC staging result in lower rates of peri- and postoperative complications than laparotomy, without negatively impacting oncologic outcomes (6). Total laparoscopic hysterectomy (TLH) with BSO is an optimal option for most patients (16), however, in non-specialized centers the management of stage I EC usually still consists of total hysterectomy and BSO followed by adjuvant radiotherapy, resulting in a high rate of morbidity and overtreatment (17). Additional co-factors (age, BMI, renal, cardiovascular status) should be taken in account during this preoperative procedure. In elderly patients possible comorbidities require more exhaustive preoperative management, but should not result in undertreatment of their gynecological cancer (18).

Moreover, setting up the strategy of the treatment could require more disciplines. Multidisciplinary should be applied because of the diverse postoperative management. Desire for childbearing, question of gender identity, sexual problems, awareness of illness, fear of recurrence are problems that should indicate the contribution of experts in other medical and non-medical fields, as well $(19,20)$.

\section{Evaluation of endometrium: role of biopsy and hysteroscopy}

During the conventional dilation and curettage (D\&C), in the majority $(60 \%)$ of the cases histological sample is obtained from less than half of the uterine cavity is curetted (21). Thus, curettage is known to miss $50-85 \%$ of focal intracavitary pathology. In $87 \%$ of women with focally growing lesions the entire or parts of the focal lesion was found to remain undetected in the uterine cavity $(22,23)$.

Endometrial samplings fail in $16-50 \%$ of cases (either technical failure or insufficient material) and in 5-20\% of these cases significant endometrial pathology is found (24-26). Endometrial sampling (with Pipelle device) has a similar reliability to curettage, although has a very limited capacity to identify polyps, and failure of getting an adequate sample is more frequent (27). Its advantage compared to curettage, that it can be performed without anaesthesia. As a non-focal pathology, atypical hyperplasia is regarded as endometrial (pre) cancer [in $17-52 \%$ an underlying cancer is found at hysterectomy (28)].

Endometrial curettage has been the traditional management for abnormal uterine bleeding, in hospitals and under general anesthesia. However, the need for admission and the risks of perforation and hemorrhage made curettage unpopular and various endometrial sampling devices were 


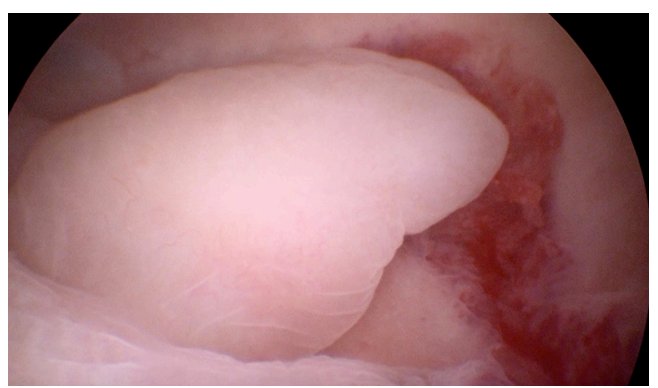

Figure 1 Hysteroscopic image of a polypoid lesion.

developed and used widely, that could be used in an outpatient setting without anesthesia (29).

According to clinical investigations, the effectiveness of hysteroscopy and targeted biopsy was superior to blind curettage in obtaining adequate endometrial samples for histological examination in any kind of uterine lesions $(\mathrm{n}=734)$ (30). In another study hysteroscopy had also better diagnostic accuracy than curettage, with significantly better sensitivity and NPV in differentiating benign and malignant endometrial lesions $(n=443)$ (31). Because of the direct visualization and the high magnification, hysteroscopy has higher accuracy for distinguishing between a normal and an abnormal endometrium, meaning the biopsies performed can be carried out in a targeted way (32). Therefore, recently curettage is almost completely replaced by hysteroscopy as a reference standard (Figure 1), both in clinical as well as in research settings (33).

In cases of hormonal therapy hysteroscopy and targeted biopsy is recommended to exclude consequent endometrial malignancy. Due to data of researches, although hormonal therapy of malignant tumors thought to cause increased incidence of other malignancies, tamoxifen therapy in breast cancer patient seems not to be associated with higher frequency of EC (34). The longer learning curve of endoscopic surgery technics may influence the selection among the available methods. However, in case of having structured, systematic training program, learning curve of minimally invasive procedures are no longer compared to the traditional methods (35).

It is also known that complications of hysteroscopy are much lower compared to blindly performed biopsy methods. Due to the visually controlled application, the rate of most frequent complication, perforation is very low. The overall success rate of diagnostic hysteroscopy was estimated at $96.9 \%$ (SD 5.2\%, range, $83-100 \%$ ) (36).

\section{Safety}

Since application of diagnostic hysteroscopy became a part of everyday gynecology, several authors proposed the hypothesis that the procedure itself might evoke the spread of malignant cells to the peritoneal cavity, by squeezing them out through the fallopian tubes (37).

Preoperative low-pressure fluid mini-hysteroscopy was found not to increase the risk of intraperitoneal transport of EC cells during the examination or the risk of pelvic recurrence at the 5-year follow-up. Neither disease-free or OS, nor recurrence rate was affected by the procedure (38).

Hysteroscopy with saline distension resulted in a statistically significant higher rate of malignant peritoneal cytology (OR, 2.89; 95\% CI, 1.48-5.64; $\mathrm{P}=0.002)$, whereas a non-significant trend for higher rate of malignant cells was observed in patients allocated to the hysteroscopy group (OR, 3.23; 95\% CI, 0.94-11.09; $\mathrm{P}=0.062$ ) when inflated media pressure reached or exceeded $100 \mathrm{mmHg}$ (39).

A large meta-analysis including almost 3,000 women diagnosed with EC, came to the conclusion that hysteroscopy could increase the number of malignant cytological findings in the peritoneal cavity. Data suggested that in cases of advanced stages of EC the application of hysteroscopy was associated with higher statistical risk of metastases. These results supported the concerns of the negative effect of the contract medium flow, which support the recommendation of hysteroscopy as a diagnostic tool mainly in cases of early stages of EC (40).

Despite published data suggesting a possible correlation between the use of hysteroscopy and the presence of malignant peritoneal cytology, there is no evidence confirming that this result is associated with hysteroscopy, reminding that-even without any diagnostic procedureendometrial cells could be detected in peritoneal cavity (41).

Although in EC the EC cells can be detected in peritoneal fluid, hysteroscopy is unlikely a significant factor in affecting intraabdominal spread of cancer cells and causing the progression of the disease resulting in higher clinical stages (42).

\section{Staging}

During preoperative work-up the assessment of the cervical involvement for staging in EC is essential. The accuracy of assessing cervical conditions was $93.2 \%$ by MRI and $55.7 \%$ by hysteroscopy (43). By using special pattern recognition 
models, sensitivity and specificity of this test were $84.6 \%$ and $81.8 \%$, respectively, with a likelihood ratio of $4: 6$ in predicting and prognosticating those women who have high-grade tumor or invasive disease (44).

Endometrial sampling performed by both pipelle device and operative hysteroscopy are of limited value to determine definitive histological type and grade with a risk of underestimated or overestimated EC risk group and thus surgical treatment (45).

In case of hysteroscopy used for the evaluation of deep ( $\geq 50 \%$ ) MI (50 studies, 3,720 patients), the pooled sensitivity was $80.7 \%$, specificity was $88.5 \%$, positive predictive value was $77.6 \%$, and NPV was $89.5 \%$, respectively (46).

Pelvic MRI (without contrast) was performed to assess the extent of $\mathrm{MI}$ ( $\mathrm{MI}<50 \%, \mathrm{MI} \geq 50 \%)$, the possible involvement of cervical stroma, status of the ovaries, and lymph nodes. Hysteroscopic excisional biopsy (HEB) was performed under general anesthesia, retrieving multiple biopsies with a $5-\mathrm{mm}$, monopolar, loop electrode. The integration of MRI and HEB showed an elevated accuracy. Sensitivity, specificity, NPV and positive predictive value were $85.0 \%, 88.5 \%, 91.9 \%$ and $79.0 \%$ in identifying lowrisk patients who do not need comprehensive surgical staging (47).

\section{Sentinel lymph node mapping}

Sentinel-lymph-node mapping has been advocated as an alternative staging technique for EC. Performing surgical staging with lymphadenectomy defines recurrence risk and helps clinicians make the decision to give adjuvant treatment to high-risk patients (48). Although most cases have negative lymph nodes, even in preoperative, supposedly extreme low-risk patients (stage I, grade I), the incidence of PAN metastases is still 4.6\% (49).

Sentinel lymph nodes marked with ICG have a high degree of diagnostic accuracy in detecting EC metastases and can safely replace lymphadenectomy in the staging of EC (10). Laparoscopic SLN mapping after injection of ICG via hysteroscopy has comparable detection rates with both radioactive tracer series and ICG injected cervically, overcoming the need for radioactive substances. Hysteroscopic injection leads to a higher mapping in the aortic area compared with cervical injection. Bilateral pelvic mapping was found in $74.5 \%$ of cases (50).

Moreover, hysteroscopy gives the opportunity even in treatment of EC in cases of having desire for fertility sparing (51), but this topic exceeds the topic of this review.

\section{Role of ultrasound in EC}

\section{Screening of asymptomatic patients}

Although transvaginal ultrasound offers easily accessible and relatively cheap option to visualize the small pelvic structure, despite intensive research it is not evidenced that ultrasound screening effectively reduces the mortality of EC. In contrast, according to cohort studies due to the high rate of false positive results, ultrasound screening strategies in asymptomatic women would result in anxiety and too many unnecessary endometrial biopsies associated with risks of surgical complications (52). Risks associated with false-positive tests include anxiety and complications from biopsies. After the completion of the United Kingdom Collaborative Trial of Ovarian Cancer Screening (UKCTOCS) including near 50,000 asymptomatic patients, it was reported, that using the double layer endometrium $5 \mathrm{~mm}$ cut-off the sensitivity was $80.5 \%$ and the specificity was $85.7 \%$ for EC diagnosed in the next 5 years. Increasing the cut off to $10 \mathrm{~mm}$, a sensitivity decreased to $54.1 \%$ and the sensitivity increased to $92.7 \%$. Based on the results, imminent biopsy is not necessary below $10 \mathrm{~mm}$, but over $5 \mathrm{~mm}$ ultrasound follow-up is recommended. However, asymptomatic menopausal women with risk factors for EC and at whom endometrial thickening and other incidental positive findings on ultrasound are present (the presence of abnormal subendometriale or endometrial vascular pattern, endometrial inhomogeneity, intravital fluid or thickened endometrium over $11 \mathrm{~mm}$ ) should be managed on a case-by-case basis (53). In addition, women at risk for EC due to a history of unopposed estrogen monotherapy, infertility with long term of chronic anovulation, late menopause, tamoxifen therapy, nulliparity, infertility or failure to ovulate, obesity, diabetes or hypertension should be informed of the risks and symptoms of EC and strongly encouraged to report any unexpected bleeding or spotting to their physicians.

\section{Endometrial polyps}

Incidental finding of polyps is frequent and with the common use and the high imaging quality the transvaginal ultrasound they are detected more often: $44.4 \%$ of the premenopausal polyps and in the $36.1 \%$ of the menopausal polyps were asymptomatic (54). Visualization of the feeding 
artery with color-Doppler improves the detection rate $(55,56)$. The majority of asymptomatic polyps detected by ultrasound and are treated surgically, although little is known about their natural behavior. They carry low risk of malignancy, hyperplasia or EC in the polyp was found in $1.7 \%$ of premenopausal and in $5.4 \%$ of postmenopausal polyps (57). Recently, analyzing the natural history by following 112 asymptomatic polyps treated expectantly (6-135 months, median 22.5) it was found, that $6.3 \%$ of polyps completely regressed and abnormal bleeding appeared in $15 \%$ independently of the growing pattern. Since that the regression rate is low and cannot be predicted, thus considering surgical treatment of polyp is a reasonable choice, especially in the menopause when polyps have higher risk of malignancy. Expert ultrasound scan may add some help to distinguish between polyps with low and high risk of malignancy. Benign polyps characteristically have smooth surface, well-defined hyperechoic borders ("bright edge"), single pedicle artery and morphologically normal endometrium adjacent to the polyp (58). In contrast, polyps with malignant potential mostly have irregular surface, multiple feeding vessels, abnormal adjacent endometrium and occasionally signs of MI (irregular endo-myometrial border) (59). Intensive studies on the detailed ultrasound features of histologically different intracavital lesions using the features recommended by the International Endometrial Tumor Analysis (IETA) group are still ongoing (60). The transvaginal image can be easily enhanced with intrauterine saline installation [saline installation sonography (SIS)], which improves the detection rate of polyps, as well as the differentiation between benign and malignant lesions. While it still remains controversial removing of endometrial polyps in asymptomatic patients, one should keep in mind that only histological evaluation could exclude focal neoplastic lesions.

\section{Ultrasonography in patients with abnormal bleeding}

One of the most investigated and debated question in the field of gynecological ultrasonography is that ultrasound could reach the NPV of endometrial biopsy, i.e., can the ultrasound examination replace the need of endometrial histology at least in a proportion of menopausal patients with abnormal bleeding. A consensus exists already from the 90-es in Europe, that based on the results of the Scandinavian and Italian multi-center studies that the risk of presence of $\mathrm{EC}$ at less than $4 \mathrm{~mm}$ double layer endometrial thickness is very minimal in patients with one episode of abnormal postmenopausal bleeding $(61,62)$. In these examinations the risk at the $4 \mathrm{~mm}$ threshold was found to be below $1 \%$, which was confirmed later by several studies. In a meta-analysis, after double-checking the clinical and imaging data of EC cases the authors concluded, that using the $3 \mathrm{~mm}$ cut off would be safer to avoid the false negative cases (63). Further, strong argument for the priority of ultrasonography over the blind biopsy, that after the followup of the patients included in the Nordic Trial it was found, that in patients with one episode of abnormal bleeding the well-documented endometrial thickness below $4 \mathrm{~mm}$ disclosed the probability on the EC better in the next ten years than negative histology in patients with endometrial thickness above $4 \mathrm{~mm}$ (64). In patients with endometrium below $4 \mathrm{~mm}$ and negative histology no EC occurred. However, in patients with recurrent abnormal bleeding the risk of EC increased to over $10 \%$ irrespective to the ultrasound finding, thus histology is mandatory in these cases. In uncertain cases or image suspicious for focal lesion the ultrasound examination can be enhanced either with intrauterine saline infusion (sonohysterography) or colorDoppler to improve the detection of abnormal endometrial structure or vascularity.

\section{Ultrasonography in EC patients}

In past decades beside MRI ultrasound is being used more intensively to support the preoperative evaluation and to select women in need for more extensive surgery for the optimal outcome. Using high resolution transvaginal probes, present imaging quality of the premium ultrasound equipment including color-Doppler and $3 \mathrm{D}$ volume acquisitions makes it possible to reach the diagnostic accuracy of MRI in the detection of the presence and the extent of various uterine pathology. According to the European Society of Gynecological Oncology (ESGO), the European Society for Medical Oncology (ESMO) and the European Society of Radiotherapy and Oncology (ESTRO), the mandatory preoperative workup of uterine cancer should include transvaginal (or transrectal) ultrasonography in addition to pelvic examination and endometrial biopsy (histological type and grade), in order to establish a preoperative FIGO staging before definitive pathological result (8). Transvaginal ultrasound is the first imaging step in patients with abnormal bleeding. In addition, color Doppler or contrast medium enhanced ultrasonography (65), preferably specialized ultrasonography, offers the possibility of evaluating the size of the tumor, ruling out ovarian disease 
and assessing MI and cervical stromal involvement (66). Despite ultrasound proved to be effective in determining the endometrial tumor margins if malignancy is present, preoperative pathological information is crucial for establishing the surgical plan. Any patients with a risk of cancer, particularly patients with postmenopausal bleeding and a hyperplastic endometrium at ultrasound, should be investigated with endometrial sampling in order to avoid uterine morcellation.

To the ESMO-ESGO-ESTRO recommendation, patients with AH/EIN or grade 1 EEC requesting fertility preservation has to be referred to specialized centers, where D\&C or preferably hysteroscopy guided biopsy has to be performed and AH/EIN or grade 1 EEC must be confirmed/diagnosed by a specialist in gynecological pathology. In addition, Pelvic MRI should be performed to establish the degree of MI and adnexal involvement, however, expert ultrasound can be considered as an alternative. In clinical stage I, grade 1 and 2 at least one of the three alternative tools should be applied to assess MI if LND is considered: good quality ultrasound by a skilled operator and/or MRI and/or intra-operative pathological examination.

The detailed ultrasound characteristics of EC according to stage and grade was also examined by the IETA group in a prospective multi-center study (67). Grayscale and color Doppler features were found to be different in high- and low-risk EC and they showed association with tumor grade and stage. These data show, that ultrasound has not reached the limits in EC staging yet. Being a reproducible, dynamic and non-invasive examination the routine application of transvaginal ultrasound in EC is recommended.

There are factors which may limit the power of ultrasound and result in under- or overestimation of myometrial and cervical invasion of the EC. According to a prospective study enrolling 210 patients and using transvaginal greyscale and power Doppler imaging according to the IETA terminology, the proportion of underestimated cases, relative to final histology was found comparable in regard to the MI (8.6\%) and the cervical invasion $(10.5 \%)$ (68). In contrast, more MI was more often overestimated with ultrasound (15.7\%) than cervical invasion (4.8\%). Interestingly, the staging error was not related to the BMI, the uterine position or to image quality. Smaller size well-differentiated ECs with thick minimum tumor-free myometrium and lower perfusion were more often underestimated, while less differentiated larger tumors with thin minimum tumor-free margin and rich perfusion were more often overestimated. The concluded that tumor size, vascular density, vessel architecture and histological grading influence the power of ultrasound staging.

With the advances of 3D ultrasound imaging the question raises, whether the 3D may add further information in order to improve the preoperative staging of EC by ultrasound. In the past years, several studies compared the power of $3 \mathrm{D}$ ultrasound and MRI in the detection of myometrial and cervical invasion of $\mathrm{EC}$, and although the result are not uniform, most of them found that MRI is not superior than 3D US $(69,70)$. Less information exists about the performance of color Doppler enhanced dynamic gray scale in relation to the MRI. Good quality ultrasound evaluation requires expertise in gynecologic disorders. The power of $2 \mathrm{D}$ and $3 \mathrm{D}$ ultrasound imaging in the EC staging was compared recently by a multicenter study, in which 15 expert examiners off-line analyzed $2 \mathrm{D}$ videos and $3 \mathrm{D}$ volumes (volume contrast imaging, VCI) of $58 \mathrm{EC}$ patients. The median accuracy for diagnosing deep (>50\%) MI was $76 \%$ with $2 \mathrm{D}$ and $69 \%$ with $3 \mathrm{D}-\mathrm{VCI}$, cervical invasion $88 \%$ with $2 \mathrm{D}$ and $86 \%$, with $3 \mathrm{D}-\mathrm{VCI}$. The interrater reliability was also better for $2 \mathrm{D}$ then for $3 \mathrm{D}$, both in the detection of deep MI and cervical involvement. The agreement between $2 \mathrm{D}$ and $3 \mathrm{D}$-VCI was weaker in diagnosing deep MI, then cervical involvement (76\% vs. $88 \%$ ). In practice it means, that in expert hands $3 \mathrm{D}-$ VI does not add more information to 2D for EC staging and detecting the cervical stromal involvement is more effective than estimating the deep MI (71).

\section{The role of MRI in the management of EC}

According to the recent NCCN guideline the role of MRI in the initial workup of EC is clearly defined. The use of pelvic MRI is recommended to assess local disease extent. Among the imaging techniques for the preoperative assessment of EC, MRI is considered to be the most accurate. The MRI appearance of EC is usually hypo- to isointense on T1-weighted images while hyperintense or heterogeneous on $\mathrm{T} 2$-weighted images compared to normal endometrium. After IV contrast injection enhancement is seen on images (72).

\section{MI}

In early stage disease the most important concern of the preoperative staging is the depth of MI. Stage IA, by the recent FIGO classification, represents tumors with myometrial tumor infiltration of less than $50 \%$. Stage 
IB indicates diseases with more than $50 \% \mathrm{MI}$ of tumor. The deep invasion of myometrium is presenting as an irregular interface and/or loss of the normal endometrium myometrium interface. For the assessment of the depth of MI sagittal T2-weighted image is a useful tool. The use of dynamic contrast-enhanced MR images improves the tumor border's delineation and helps to assess the affected depth of invasion in the myometrium, since the endometrial tissue is less enhanced than myometrium (72). Goel et al. reported in their prospective, that the sensitivity and specificity of MRI for assessment the MI were $75 \%$ and $73 \%$ respectively, the overall accuracy was $74.14 \%$ (73). Additionally, the use of diffusion weighted imaging (DWI) is increasing the assessment of depth of MI. Moreover, the DWI can be helpful in patients who cannot receive gadolinium based contrast agent administered intravenously (72).

\section{Cervical involvement}

The presence of cervical stromal invasion without spreading beyond the uterus is an important factor representing stage II disease. Sensitivity of MRI is poor in $37.5 \%$ and $50 \%$ respectively) predicting extrauterine extension and cervical invasion but specificity is excellent (92\% and 100\% respectively). Goel's study shows that the preoperative MRI not only has high sensitivity and specificity in prediction of MI and but it is very useful modality to assess lymph node involvement. On the contrary, prediction of cervical involvement or extrauterine spread, it is not sensitive enough. Based on these results, the use of $3 \mathrm{D}$-Ultrasound in the preoperative assessment of myometrial and cervical infiltration of tumor can be a useful tool (73).

Yildirim and co-workers examined the diagnostic accuracy of 3D-US for detecting deep myometrial, lower uterine segment and cervical invasion, and these were found to be $87.5 \%, 80 \%$ and $85 \%$, respectively. The same results for MRI were $75 \%, 65 \%$ and $70 \%$ in their study respectively. According to their results the $3 \mathrm{D}-\mathrm{US}$ had higher sensitivity, specificity, negative and positive predictive value and accuracy than MRI. The combination of these two imaging techniques increased the sensitivity, but decreased the specificity and the accuracy. 3D-US was an easier, cheaper and effective imaging method for triaging the early stage EC (69).

\section{Lymph node metastasis}

The stage IIIC according to recent FIGO classification, covers the tumors with lymph node metastasis. This stage is divided into two subgroups, in stage IIIC1 there is pelvic while in stage IIIC2 para-aortic lymph node involvement. The presence of nodal involvement has a good correlation with the depth of MI. In cases with stage IA disease the incidence of lymph node metastases is $3 \%$, but if $\mathrm{MI}$ is greater than $50 \%$ the incidence of metastatic lymph nodes is $46 \%$ (72). In Goel's prospective study the MRI had a good sensitivity $(88.64 \%)$, a moderate specificity $(66.67 \%)$, to predict the lymph node spreading, the overall accuracy was $86 \%$ (73). Arian et al. examined the accuracy of diffusionweighted MRI (DW-MRI) to predict the pelvic lymph node metastases (74). In their retrospective study 33 women were enrolled with histologically confirmed EC. According to this study, the DW-MRI had a sensitivity of $80.6 \%$, a specificity of $100 \%$ for predicting lymph node involvement and the overall accuracy was $87.5 \%$ in discrimination between a metastatic and non-metastatic pattern. DW-MRI was a valuable tool for differentiating between metastatic and benign lymph nodes in EC patients (74). In a recent study the utility of three-dimensional MRI (3D-MRI) was evaluated. The relation between tumor volume measured with 3D-MRI and lymph node metastasis was examined in patients with EC. Multivariate analysis revealed that tumor volume was an independent risk factor for lymphatic metastasis [hazard ratio (HR) 12.7, 95\% CI: 1.06-154]. The potential cut-off value of tumor volume was $12.79 \mathrm{~cm}^{3}$ (sensitivity: 0.821 ; specificity: 0.744$)$ (75).

\section{Role of functional MRI}

The histological tumor grade is a risk factor of nodal involvement, therefore preoperative accurate determination is a key factor in defining necessity of lymphadenectomy. Tanaka $e t$ al. in their work examined ninety-one patient's apparent diffusion coefficient (ADC) on DW-MRI and compared to the frozen section (FS) diagnosis made at hysterectomy. The diagnostic accuracy of DWI and FS was compared in prediction of the tumor grade, based on final histological reports. The high-grade (endometrioid G3) tumors had lower ADC values than low-grade (endometrioid G1/2) tumors. There was no significant difference in the true positive rates of $\mathrm{ADC}$ values and $\mathrm{FS}$ s in the prediction of high-grade tumors ( $73.3 \%$ vs. $66.7 \%, \mathrm{P}=0.7)$, however, in the prediction of low grade tumors the true negative rate of ADC values was significantly lower than that of the FSs (64.5\% vs. 98.7\%, $\mathrm{P}=0.01)$. In conclusion the FS diagnosis was more accurate to predict high-grade tumors than DW- 
MRI (76).

In Song's study the T2-weighted image (T2WI) and conventional diffusion-weighted image (cDWI) was compared in the sensitivity of qualitative diagnosis and the accuracy was examined in prediction of tumor size (TS). The TS of T2WI was in accordance with the actual size for all ECs. DWI had a high sensitivity in detecting the discrepancy of pathology between different tissues, so it had an advantage in diagnosing cancer in an early stage. DWI was better than T2WI in the qualitative diagnosis, and T2WI was better than DWI at tumor size measurement (77).

To summarize, the use of MRI in the preoperative workup is recommended, for in the prediction of $\mathrm{MI}$ and lymph node involvement it has high sensitivity and high specificity (73), although it has moderate or low sensitive to predict cervical involvement or extrauterine spread. Recently, the MRI seems to be a moderately sensitive and specific tool for preoperative staging of EC. According to the above mentioned data, we need further refinements in the use of MRI during preoperative workup of EC and further investigations to find the best modality of this useful tool (73). These updated ESUR Female Pelvis Imaging working group guidelines and the recent ESMO guidelines represents the most recent developments in this field (78).

\section{Biomarkers in EC}

Obtaining of a representative preoperative sample of the EC (preferably via hysteroscopy as explained above) is essential for the proper preoperative histological evaluation of the tumor. The classic histological classification of EC differentiates the much more common type I tumors (80-90\%) (endometrioid adenocarcinoma) and type II tumors (10-20\%) comprising non-endometrioid subtypes (serous, clear cell, undifferentiated carcinoma and malignant mixed Müllerian tumor) (79). This has become determining factor besides the spread of the tumor in the operative management (including possible fertility sparing treatment and extent of staging procedures) and adjuvant treatment according to the present guidelines (8). Unfortunately, inter-observer disagreement regarding histology is a common phenomenon: in the central pathological reevaluation of the PORTEC-3 trial there was common interobserver disagreement for histological type (34\%), grade (19\%) and stromal involvement (27\%) (80). Molecular data mostly supports this dual classification: the most common gene alterations in type I tumors are of the PTEN, KRAS, CTNNB1, PIK3 and MLH1 promoter hypermethylation while in type 2 cancers the TP53 mutations are most frequent, however some endometrioid carcinomas are also associated with mutated TP53 and therefore have extreme genetic instability, with unfavorable prognosis (81). The Cancer Genome Atlas Research Network identified 4 molecular subtypes, which are the following: (I) POLE (ultra-mutated) tumors; (II) MSI tumors; (III) copy-number high tumors with TP53 mutations; (IV) remaining group without the upper alterations (8).

The molecular analysis of the PORTEC 1 and 2 trials showed, that POLE mutations were associated with high grade, although are capable of identifying a subgroup of these tumors which are associated with a very good prognosis: in these trials none of the POLE mutant ECs have recurred, while $30.9 \%$ of the POLE-wild type ones did (82). This biomarker might be useful in the future to avoid over-treatment of approximately $10 \%$ of clinically good prognostic high-grade tumors. Inspired by the TCGA (which required fresh-frozen samples) a classification called ProMisE (Proactive Molecular Risk Classifier for Endometrial Cancer) was developed, which identifies 4 subtypes based on, but not equivalent to the TCGA ones: MMR-D (mismatch repair deficient), POLE, p 53 abnormal and p53 wild type (83). Very importantly applicability of the molecular subtypes of the diagnostic specimen (e.g., biopsies) showed high reproductivity with the final histologies (73). The p53 abn subtype showed aggressive characteristics (MI, positive nodes, LVSI) having been classified as ESMO 2016 high risk types in $87 \%$. The second most aggressive type was MMR-D. The largest subtype was p53 wild type (usually low-grade, low-stage, and endometrioid in 99\%) classified as ESMO "low risk" in $62 \%$ (83). The patients with POLE subtype had excellent prognosis in this series as well, and have been confirmed by other independent studies in the literature $(84,85)$.

Another very promising example for the use of biomarkers in EC is the detection of aberrant telomere length in circulating cell free DNA (cfDNA). Benati and colleagues described a technique for the measurement of relative telomere length (RTL) in cfDNA for the detection of endometrioid EC, and using ROC curve analysis it had a sensitivity of $80 \%$ at a specificity of $80.65 \%$, regardless of the stage or grade of the disease. This might be a useful tool in the future for the early detection of EC especially in highrisk groups of patients (e.g., those with atypical endometrial hyperplasia) (86). The use of molecular biomarkers helps to decrease the inter-observer variability, the poor correlation of grade ad histotype between the diagnostic specimen 
and results of the final histology $(45,87)$, which might be a frequently seen feature in case of high grade tumors $(88,89)$. Hysteroscopy helps us obtaining a representative sample in the diagnosis of $\mathrm{EC}$, and with the use of biomarkers we can overcome the histological "mimics". The use of POLE biomarker proves to be a promising tool in the future to avoid over-treatment of approximately $10 \%$ of clinically good prognostic high-grade tumors. The incorporation of molecular characteristics into EC management (90) should influence our treatment strategies in the near future.

\section{Summary}

In conclusion, all MRI, HEB and high resolution $2 \mathrm{D}$ or $3 \mathrm{D}$ ultrasound performed by expert operator are considered to add valuable information for preoperative staging of EC. The most preferred method is still MRI over the others, but guidelines are not uniform in the positioning of the imaging modalities, and since their performances are constantly improving due to rapid technical development, their diagnostic position may change. To the present it is difficult to establish the superiority of any of them over the other, all may add valuable information for the preoperative staging and centers are recommended to use them according to the local availability, quality and experience. We always have to keep in mind, that the main goal (and not more) of the preoperative assessment is to distinguish between the low-risk (stage I, grade 1 and 2) and the more advanced $\mathrm{EC}$, or raise the suspicion of non-endometrioid cancer, which guides the optimal management plan. Developing an integrated diagnostic work-up would, therefore, be greatly beneficial and could represent the best chance to optimize surgical management, avoiding over- or undertreatment of EC patients. Further studies on large cohorts and with great statistical power may answer which is the best integrated diagnostic work-up at present to stratify patients for lowand high-risk on lymph node involvement.

Accurate preoperative assessment of the lymph nodes would ideally identify those patients with advanced stage disease, who might benefit from more extensive surgical procedures and adjuvant therapies. However, present imaging modalities have higher accuracy in the detection of MI and cervical involvement, than lymph nodes metastases.

Additional CT scan can complement this disadvantage of the US to disclose lymph node pathology, while ultrasound in expert hands can safely evaluate deep MI, or cervical involvement. This ultrasound plus CT scan setting could increase the availability to preoperative imaging compared to MRI, since the later being a far more time-consuming procedure, limiting its availability at a large number of healthcare systems. CT imaging of the whole abdominal region is essential, since in the absence of confirmed pelvic lymph node metastases, rarely paraaortic lymph node metastases may be present.

The use of biomarkers could be beneficial in decreasing inter-observer variability between the histology of the diagnostic specimen and the final operative sample, as well as to avoid overtreatment in a part of the high-grade tumors with excellent prognosis. Extended surgery, including paraaortic lymphadenectomy has a significant morbidity, and with the future selection of cases it can be safely avoided, we can decrease complications without compromising oncological safety. In the future, the importance of hysteroscopy guided sampling may increase to gain a representative sample for biomarker detection.

\section{Acknowledgments}

Funding: None.

\section{Footnote}

Provenance and Peer Review: This article was commissioned by the Guest Editor (Antonio Simone Laganà) for the series "Endometrial Cancer" published in Translational Cancer Research. The article was sent for external peer review organized by the Guest Editor and the editorial office.

Conflicts of Interest: All authors have completed the ICMJE uniform disclosure form (available at http://dx.doi. org/10.21037/tcr-20-2068). The series "Endometrial Cancer" was commissioned by the editorial office without any funding or sponsorship. The authors have no other conflicts of interest to declare.

Ethical Statement: The authors are accountable for all aspects of the work in ensuring that questions related to the accuracy or integrity of any part of the work are appropriately investigated and resolved.

Open Access Statement: This is an Open Access article distributed in accordance with the Creative Commons Attribution-NonCommercial-NoDerivs 4.0 International License (CC BY-NC-ND 4.0), which permits the noncommercial replication and distribution of the article with the strict proviso that no changes or edits are made and the 
original work is properly cited (including links to both the formal publication through the relevant DOI and the license). See: https://creativecommons.org/licenses/by-nc-nd/4.0/.

\section{References}

1. Bray F, Dos Santos Silva I, Moller H, et al. Endometrial cancer incidence trends in Europe: underlying determinants and prospects for prevention. Cancer Epidemiol Biomarkers Prev 2005;14:1132-42.

2. Andrijasevic S, Dotlic J, Arsenovic N, et al. Differences in endometrial carcinoma presentations and characteristics in pre- and postmenopausal women. Srp Arh Celok Lek 2019;147:692-8.

3. Creutzberg CL, van Putten WL, Koper PC, et al. Surgery and postoperative radiotherapy versus surgery alone for patients with stage- 1 endometrial carcinoma: multicentre randomised trial. PORTEC Study Group. Post Operative Radiation Therapy in Endometrial Carcinoma. Lancet 2000;355:1404-11.

4. Valsecchi L, Mangili G, Frigerio L, et al. Reliability of preoperative evaluation of prognostic factors in endometrial carcinoma. Int J Gynaecol Obstet 1997;59:35-9.

5. Casarin J, Bogani G, Serati M, et al. Presence of Glandular Cells at the Preoperative Cervical Cytology and Local Recurrence in Endometrial. Int J Gynecol Pathol 2020;39:522-8.

6. Walker JL, Piedmonte MR, Spirtos NM, et al. Laparoscopy compared with laparotomy for comprehensive surgical staging of uterine cancer: Gynecologic Oncology Group Study LAP2. J Clin Oncol 2009;27:5331-6.

7. Franchi M, Garzon S, Zorzato PC, et al. PET-CT Scan in the Preoperative workup of early stage intermediate- and high-risk endometrial cancer. Minim Invasive Ther Allied Technol 2020;29:232-9.

8. Colombo N, Creutzberg C, Amant F, et al. ESMOESGO-ESTRO Consensus Conference on Endometrial Cancer: diagnosis, treatment and follow-up Ann Oncol 2016;27:16-41.

9. Cibula D, Pötter R, Planchamp F, et al. The European Society of Gynaecological Oncology/European Society for Radiotherapy and Oncology/European Society of Pathology Guidelines for the Management of Patients With Cervical Cancer. Int J Gynecol Cancer 2018;28:641-55.

10. Rossi EC, Kowalski LD, Scalici J, et al. A comparison of sentinel lymph node biopsy to lymphadenectomy for endometrial cancer staging (FIRES trial): a multicentre, prospective, cohort study. Lancet Oncol 2017;18:384-92.

11. Persson J, Salehi S, Bollino M, et al. Pelvic Sentinel lymph node detection in High-Risk Endometrial Cancer (SHREC-trial)-the final step towards a paradigm shift in surgical staging. Eur J Cancer 2019;116:77 85.

12. NCCN Clinical Practice Guideline in Oncology. Uterine neoplasms. NCCN Evidence Blocks. Version 1.2020-March 16, 2020.

13. May K, Bryant A, Dickinson HO, et al. Lymphadenectomy for the management of endometrial cancer. Cochrane Database Syst Rev 2010;(1):CD007585.

14. Seagle BL, Alexander AL, Lantsman T, et al. Prognosis and treatment of positive peritoneal cytology in early endometrial cancer: matched cohort analyses from the National Cancer Database. Am J Obstet Gynecol 2018;218:329.e1-329.e15.

15. Wright JD, Burke WM, Tergas AI, et al. Comparative Effectiveness of Minimally Invasive Hysterectomy for Endometrial Cancer. J Clin Oncol 2016;34:1087-96.

16. Cusimano MC, Simpson AN, Dossa F, et al. Laparoscopic and robotic hysterectomy in endometrial cancer patients with obesity: a systematic review and meta-analysis of conversions and complications. Am J Obstet Gynecol 2019;221:410-428.e19.

17. ASTEC/EN.5 Study Group, Blake P, Swart AM, et al. Adjuvant external beam radiotherapy in the treatment of endometrial cancer (MRC ASTEC and NCIC CTG EN.5 randomised trials): pooled trial results, systematic review, and meta-analysis. Lancet 2009;373:137-46.

18. Vitale SG, Capriglione S, Zito G, et al. Management of endometrial, ovarian and cervical cancer in the elderly: current approach to a challenging condition. Arch Gynecol Obstet 2019;299:299-315.

19. Rosa VL, Garzon S, Gullo G, et al. Fertility preservation in women affected by gynaecological cancer: the importance of an integrated gynaecological and psychological approach. Ecancermedicalscience 2020;14:1035.

20. Jeppesen MM, Mogensen O, Dehn P, et al. Needs and priorities of women with endometrial and cervical cancer. J Psychosom Obstet Gynaecol 2015;36:122-32.

21. Stock RJ, Kanbour A. Prehysterectomy curettage. Obstet Gynecol 1975;45:537-41.

22. Epstein E, Ramirez A, Skoog L, et al. Dilatation and curettage fails to detect most focal lesions in the uterine cavity in women with postmenopausal bleeding. Acta Obstet Gynecol Scand 2001;80:1131-6.

23. Gebauer G, Hafner A, Siebzehnrübl E, et al. Role of 
hysteroscopy in detection and extraction of endometrial polyps: results of a prospective study. Am J Obstet Gynecol 2001;184:59-63.

24. Farrell T, Jones N, Owen P, et al. The significance of an 'insufficient' Pipelle sample in the investigation of post-menopausal bleeding. Acta Obstet Gynecol Scand 1999;78:810-2.

25. Visser NC, Breijer MC, Herman MC, et al. Factors attributing to the failure of endometrial sampling in women with postmenopausal bleeding. Acta Obstet Gynecol Scand 2013;92:1216-22.

26. van Doorn HC, Opmeer BC, Burger CW, et al. Inadequate office endometrial sample requires further evaluation in women with postmenopausal bleeding and abnormal ultrasound results. Int J Gynaecol Obstet 2007;99:100-4.

27. Terzic MM, Aimagambetova G, Terzic S, et al. Current role of Pipelle endometrial sampling in early diagnosis of endometrial cancer. Transl Cancer Res 2020;9:7716-24.

28. Trimble CL, Kauderer J, Zaino R, et al. Concurrent endometrial carcinoma in women with a biopsy diagnosis of atypical endometrial hyperplasia: a Gynecologic Oncology Group study. Cancer 2006;106:812-9.

29. Narice BF, Delaney B, Dickson JM. Endometrial sampling in low-risk patients with abnormal uterine bleeding: a systematic review and meta-synthesis. BMC Fam Pract 2018;19:135.

30. Bedner R, Rzepka-Górska I. Hysteroscopy with directed biopsy versus dilatation and curettage for the diagnosis of endometrial hyperplasia and cancer in perimenopausal women. Eur J Gynaecol Oncol 2007;28:400-2.

31. Ceci O, Bettocchi S, Pellegrino A, et al. Comparison of hysteroscopic and hysterectomy findings for assessing the diagnostic accuracy of office hysteroscopy. Fertil Steril 2002;78:628-31.

32. Loverro G, Bettocchi S, Cormio G, et al. Transvaginal sonography and hysteroscopy in postmenopausal uterine bleeding. Maturitas 1999;33:139-44.

33. Clark TJ, Voit D, Gupta JK, et al. Accuracy of hysteroscopy in the diagnosis of endometrial cancer and hyperplasia: a systematic quantitative review. JAMA 2002;288:1610-21.

34. Chiofalo B, Mazzon I, Di Angelo Antonio S, et al. Hysteroscopic Evaluation of Endometrial Changes in Breast Cancer Women with or without Hormone Therapies: Results from a Large Multicenter Cohort Study. J Minim Invasive Gynecol 2020;27:832-9.

35. Hiemstra E, Kolkman W, le Cessie S, et al. Are minimally invasive procedures harder to acquire than conventional surgical procedures? Gynecol Obstet Invest 2011;71:268-73.

36. van Dongen H, de Kroon CD, Jacobi CE, et al. Diagnostic hysteroscopy in abnormal uterine bleeding: a systematic review and meta-analysis. BJOG 2007;114:664-75.

37. Dueholm M, Hjorth IM, Secher P, et al. Structured Hysteroscopic Evaluation of Endometrium in Women With Postmenopausal Bleeding. J Minim Invasive Gynecol 2015;22:1215-24.

38. Cicinelli E, Tinelli R, Colafiglio G, et al. Risk of long-term pelvic recurrences after fluid minihysteroscopy in women with endometrial carcinoma: a controlled randomized study. Menopause 2010;17:511-5.

39. Polyzos NP, Mauri D, Tsioras S, et al. Intraperitoneal dissemination of endometrial cancer cells after hysteroscopy: a systematic review and meta-analysis. Int J Gynecol Cancer 2010;20:261-7.

40. Chang YN, Zhang Y, Wang YJ, et al. Effect of hysteroscopy on the peritoneal dissemination of endometrial cancer cells: a meta-analysis. Fertil Steril 2011;96:957-61.

41. Selvaggi L, Cormio G, Ceci O, et al. Hysteroscopy does not increase the risk of microscopic extrauterine spread in endometrial carcinoma. Int J Gynecol Cancer 2003;13:223-7.

42. Revel A, Tsafrir A, Anteby SO, et al. Does hysteroscopy produce intraperitoneal spread of endometrial cancer cells? Obstet Gynecol Surv 2004;59:280-4.

43. Xu G, Wang D, Ling X, et al. Diagnostic Value of Assessment of Cervical Involvement in Early-Stage Endometrial Adenocarcinoma: Comparison of Magnetic Resonance Imaging (MRI) Versus Hysteroscopy. Med Sci Monit 2018;24:7952-7.

44. Su H, Pandey D, Liu LY, et al. Pattern Recognition to Prognosticate Endometrial Cancer: The Science Behind the Art of Office Hysteroscopy-A Retrospective Study. Int J Gynecol Cancer 2016;26:705-10.

45. Phelippeau J, Canlorbe G, Bendifallah S, et al. Preoperative diagnosis of tumor grade and type in endometrial cancer by pipelle sampling and hysteroscopy: Results of a French study. Surg Oncol 2016;25:370-7.

46. Luomaranta A, Leminen A, Loukovaara M. Magnetic resonance imaging in the assessment of high-risk features of endometrial carcinoma: a meta-analysis. Int J Gynecol Cancer 2015;25:837-42.

47. Cignini P, Vitale SG, Laganà AS, et al. Preoperative workup for definition of lymph node risk involvement in early stage endometrial cancer: 5-year follow-up. Updates Surg 
2017;69:75-82.

48. SGO Clinical Practice Endometrial Cancer Working Group, Burke WM, Orr J, et al. Endometrial cancer: a review and current management strategies: part I. Gynecol Oncol 2014;134:385-92.

49. Yoon JH, Yoo SC, Kim WY, Para-aortic lymphadenectomy in the management of preoperative grade 1 endometrial cancer confined to the uterine corpus. Ann Surg Oncol 2010;17:3234-40.

50. Martinelli F, Ditto A, Bogani G, et al. Laparoscopic Sentinel Node Mapping in Endometrial Cancer After Hysteroscopic Injection of Indocyanine Green. J Minim Invasive Gynecol 2017;24:89-93.

51. Vitale SG, Rossetti D, Tropea A, et al. Fertility sparing surgery for stage IA type I and G2 endometrial cancer in reproductive-aged patients: evidence-based approach and future perspectives. Updates Surg 2017;69:29-34.

52. Dreisler E, Sorensen SS, Ibsen PH, et al. Value of endometrial thickness measurement for diagnosing focal intrauterine pathology in women without abnormal uterine bleeding. Ultrasound Obstet Gynecol 2009;33:344-8.

53. Jacobs I, Gentry-Maharaj A, Burnell M, et al. Sensitivity of transvaginal ultrasound screening for endometrial cancer in postmenopausal women: a case-control study within the UKCTOCS cohort. Lancet Oncol 2011;12:38-48.

54. Hassa H, Tekin B, Senses T, et al. Are the site, diameter, and number of endometrial polyps related with symptomatology? Am J Obstet Gynecol 2006;194:718-21.

55. Timmerman D, Verguts J, Konstantinovic ML, et al. The pedicle artery sign based on sonography with color Doppler imaging can replace second-stage tests in women with abnormal vaginal bleeding. Ultrasound Obstet Gynecol 2003;22:166-71.

56. Jakab A, Ovári L, Juhász B, et al. Detection of feeding artery improves the ultrasound diagnosis of endometrial polyps in asymptomatic patients. Eur J Obstet Gynecol Reprod Biol 2005;119:103-7.

57. Lee SC, Kaunitz AM, Sanchez-Ramos L, et al. The oncogenic potential of endometrial polyps: a systematic review and meta-analysis. Obstet Gynecol 2010;116:1197-205.

58. Valentin L. Imaging techniques in the management of abnormal vaginal bleeding in non-pregnant women before and after menopause. Best Pract Res Clin Obstet Gynaecol 2014;28:637-54.

59. Alcázar JL, Castillo G, Mínguez JA, et al. Endometrial blood flow mapping using transvaginal power Doppler sonography in women with postmenopausal bleeding and thickened endometrium. Ultrasound Obstet Gynecol 2003;21:583-8.

60. Leone FP, Timmerman D, Bourne T, et al. Terms, definitions and measurements to describe the sonographic features of the endometrium and intrauterine lesions: a consensus opinion from the International Endometrial Tumor Analysis (IETA) group. Ultrasound Obstet Gynecol 2010;35:103-12.

61. Karlsson B, Granberg S, Wikland M, et al. Transvaginal ultrasonography of the endometrium in women with postmenopausal bleeding - a Nordic multicenter study. Am J Obstet Gynecol 1995;172:1488-94.

62. Ferrazzi E, Torri V, Trio D, et al. Sonographic endometrial thickness: a useful test to predict atrophy in patients with postmenopausal bleeding. An Italian multicenter study. Ultrasound Obstet Gynecol 1996;7:315-21.

63. Timmermans A, Opmeer BC, Khan KS, et al. Endometrial thickness measurement for detecting endometrial cancer in women with postmenopausal bleeding: a systematic review and meta-analysis. Obstet Gynecol 2010;116:160-7.

64. Gull B, Karlsson B, Milsom I, et al. Can ultrasound replace dilation and curettage? A longitudinal evaluation of postmenopausal bleeding and transvaginal sonographic measurement of the endometrium as predictors of endometrial cancer. Am J Obstet Gynecol 2003;188:401-8.

65. Eriksson LS, Lindqvist PG, Flöter Rådestad A, et al. Transvaginal ultrasound assessment of myometrial and cervical stromal invasion in women with endometrial cancer: interobserver reproducibility among ultrasound experts and gynecologists. Ultrasound Obstet Gynecol 2015;45:476-82.

66. Epstein E, Blomqvist L. Imaging in endometrial cancer. Best Pract Res Clin Obstet Gynaecol 2014;28:721-39.

67. Epstein E, Fischerova D, Valentin L, et al. Ultrasound characteristics of endometrial cancer as defined by International Endometrial Tumor Analysis (IETA) consensus nomenclature: prospective multicenter study. Ultrasound Obstet Gynecol 2018;51:818-28.

68. Fischerova D, Frühauf F, Zikan M, et al. Factors affecting sonographic preoperative local staging of endometrial cancer. Ultrasound Obstet Gynecol 2014;43:575-85.

69. Yildirim N, Saatli B, Kose S, et al. Predictability of myometrial, lower uterine segment and cervical invasion with $3 \mathrm{D}$ transvaginal ultrasonography and magnetic resonance imaging in endometrial cancer patients: a prospective cohort study. Med Ultrason 2018;20:348-54.

70. Saarelainen SK, Kööbi L, Järvenpää R, et al. The preoperative assessment of deep myometrial invasion by 
three-dimensional ultrasound versus MRI in endometrial carcinoma. Acta Obstet Gynecol Scand 2012;91:983-90.

71. Green RW, Valentin L, Alcazar JL, et al. Endometrial cancer off-line staging using two-dimensional transvaginal ultrasound and three-dimensional volume contrast imaging: Intermethod agreement, interrater reliability and diagnostic accuracy. Gynecol Oncol 2018;150:438-45.

72. Faria SC, Devine CE, Rao B, et al. Imaging and Staging of Endometrial Cancer. Semin Ultrasound CT MR 2019;40:287-94.

73. Goel G, Rajanbabu A, Sandhya CJ, et al. A Prospective Observational Study Evaluating the Accuracy of MRI in Predicting the Extent of Disease in Endometrial Cancer. Indian J Surg Oncol 2019;10:220-4.

74. Arian A, Easa AM, Arab-Ahmadi M. Diagnostic value of diffusion-weighted magnetic resonance imaging in discriminating between metastatic and non-metastatic pelvic lymph nodes in endometrial cancer. Acta Radiol 2020;61:1580-6.

75. Tanase Y, Takahama J, Kawaguchi R, et al. Analysis of Risk Factors for Lymphatic Metastasis in Endometrial Carcinoma and Utility of Three-Dimensional Magnetic Resonance Imaging in Gynecology. World J Oncol 2018;9:74-9.

76. Tanaka T, Terai Y, Fujiwara S, et al. Preoperative diffusion-weighted magnetic resonance imaging and intraoperative frozen sections for predicting the tumor grade in endometrioid endometrial cancer. Oncotarget 2018;9:36575-84.

77. Song Y, Shang H, Ma Y, et al. Can conventional DWI accurately assess the size of endometrial cancer? Abdom Radiol (NY) 2020;45:1132-40.

78. Nougaret S, Horta M, Sala E, et al. Endometrial Cancer MRI staging: Updated Guidelines of the European Society of Urogenital Radiology. Eur Radiol 2019;29:792-805.

79. American College of Obstetricians and Gynecologists. ACOG practice bulletin, clinical management guidelines for obstetrician-gynecologists, number 65, August 2005: management of endometrial cancer. Obstet Gynecol 2005;106:413-25.

Cite this article as: Török P, Krasznai Z, Molnár S, Lampé R, Jakab A. Preoperative assessment of endometrial cancer. Transl Cancer Res 2020;9(12):7746-7758. doi: 10.21037/tcr-20-2068
80. de Boer SM, Wortman BG, Bosse T, et al. Clinical consequences of upfront pathology review in the randomised PORTEC-3 trial for high-risk endometrial cancer. Ann Oncol 2018;29:424-30.

81. Kandoth C, McLellan MD, Vandin F, et al. Mutational landscape and significance across 12 major cancer types. Nature 2013;502:333-9.

82. Church DN, Stelloo E, Nout RA, et al. Prognostic significance of POLE proofreading mutations in endometrial cancer. J Natl Cancer Inst 2014;107:402.

83. Kommoss S, McConechy MK, Kommoss F, et al. Final validation of the ProMisE molecular classifier for endometrial carcinoma in a large population-based case series. Ann Oncol 2018;29:1180-8.

84. Talhouk A, McConechy MK, Leung S, et al. Confirmation of ProMisE: a simple, genomics-based clinical classifier for endometrial cancer. Cancer 2017;123:802-13.

85. Meng B, Hoang LN, McIntyre JB, et al. POLE exonuclease domain mutation predicts long progressionfree survival in grade 3 endometrioid carcinoma of the endometrium. Gynecol Oncol 2014;134:15-9.

86. Benati M, Montagnana M, Danese E, et al. Aberrant Telomere Length in Circulating Cell-Free DNA as Possible Blood Biomarker with High Diagnostic Performance in Endometrial Cancer. Pathol Oncol Res 2020;26:2281-9.

87. Talhouk A, Hoang LN, McConechy MK, et al. Molecular classification of endometrial carcinoma on diagnostic specimens is highly concordant with final hysterectomy: earlier prognostic information to guide treatment. Gynecol Oncol 2016;143:46-53.

88. Han G, Sidhu D, Duggan MA, et al. Reproducibility of histological cell type in high-grade endometrial carcinoma. Mod Pathol 2013;26:1594-604.

89. Clarke BA, Gilks CB. Endometrial carcinoma: controversies in histopathological assessment of grade and tumour cell type. J Clin Pathol 2010;63:410-5.

90. Vermij L, Smit V, Nout R, et al. Incorporation of molecular characteristics into endometrial cancer management. Histopathology 2020;76:52-63. 